\title{
CETEM
}

Ministério da Ciência e Tecnologia

Coordenação de Processos Metalúrgicos e Ambientais

\section{Ensaio de Comportamento com Minhocas (Eisenia fetida) para Avaliação de Áreas Contaminadas: Resultados Preliminares para Contaminação por Hidrocarbonetos}

Cristina Lucia Sisinno

Josino Moreira

FIOCRUZ

Martha Bulus

FEEMA

Andréa Rizzo

CETEM

CT2006-047-00 - Comunicação Técnica ao Journal of the Brazilian Society of Ecotoxicology (ISSN 1809-8401), v.1, n. 2, 2006, p 41-44. 


\title{
Ensaio de Comportamento com Minhocas (Eisenia fetida) para Avaliação de Áreas Contaminadas: Resultados Preliminares para Contaminação por Hidrocarbonetos
}

\author{
C. L. S. Sisinno, ${ }^{1 *}$ M. R. M. Bulus, ${ }^{2}$ A. C. Rizzo ${ }^{3}$ \& J. C. Moreira ${ }^{1}$ \\ ${ }^{1}$ Fundação Oswaldo Cruz (FIOCRUZ), Escola Nacional de Saúde Pública - DSSA, \\ Rua Leopoldo Bulhões, 1480, 5o andar, Manguinhos, CEP 21041-210, Rio de Janeiro, RJ \\ ${ }^{2}$ Fundação Estadual de Engenharia do Meio Ambiente (FEEMA), Setor de Ecotoxicologia, \\ Av. Salvador Allende, 5.500, Jacarepaguá, CEP 22780-160, Rio de Janeiro, RJ \\ ${ }^{3}$ Centro de Tecnologia Mineral (CETEM), Coordenação de Desenvolvimento Sustentável, Av. Ipê, 900, \\ Ilha do Fundão, CEP21941-590, Rio de Janeiro, RJ
}

\begin{abstract}
RESUMO
Os problemas oriundos de áreas contaminadas vêm crescendo cada vez mais no Brasil. Essas áreas podem ser representadas por postos de serviços, locais de disposição inadequada de resíduos sólidos, indústrias desativadas, etc. e muitas vezes se encontram nas proximidades de ecossistemas, causando impactos negativos. A avaliação da contaminação dessas áreas tem sido realizada apenas com o auxílio de parâmetros químicos, sem a inserção da avaliação ecotoxicológica. O objetivo deste trabalho foi iniciar ensaios preliminares segundo o draft da ISO que descreve um ensaio de comportamento para testar a qualidade de solos e a toxicidade de substâncias químicas com o organismo Eisenia fetida (minhoca). Esse ensaio pode ser aplicado como um método rápido para determinar a biodisponibilidade de substâncias químicas ou de contaminantes no solo para $E$. fetida, em que o comportamento de fuga é usado como indicador. Com base nos resultados, pode ser decidido se o ensaio de reprodução com a mesma espécie deve ou não ser realizado. Amostras de áreas contaminadas com diferentes concentrações de hidrocarbonetos provindas de uma garagem de ônibus, um posto de serviços e uma área com derramamento de óleo foram analisadas por meio desse ensaio. Os resultados da amostra originada da garagem de ônibus mostraram que $96 \%$ dos organismos fugiram da seção que continha a amostra contaminada para a seção onde estava o solo controle, indicando que a amostra pode ser considerada tóxica, apresentando sua função de habitat limitada. A toxicidade dessa amostra foi posteriormente confirmada pelos ensaios de letalidade e reprodução. Os resultados preliminares indicaram que o ensaio de comportamento foi um indicador rápido da toxicidade da amostra e pode ser aplicado como complementação da avaliação de áreas contaminadas por hidrocarbonetos.
\end{abstract}

Palavras-chave: ecotoxicologia terrestre, minhoca, Eisenia fetida, áreas contaminadas, hidrocarbonetos.

\section{ABSTRACT \\ Avoidance test using earthworms (Eisenia fetida) for contaminated sites evaluation: preliminary results for hydrocarbon contamination}

Problems related to contaminated sites are increasing in Brazil. These sites can be represented by gas station, waste disposal areas, abandoned industrial facilities, etc. and in general are located in the neighborhood of important ecosystems, being cause of negative impacts. Evaluation of contaminated sites in Brazil has been done using only chemical parameters, without ecotoxicological studies. The aim of this study was initiate preliminary tests according to the draft of ISO that

\footnotetext{
*Corresponding author: Cristina L. S. Sisinno, e-mail: cristina.sisinno@uol.com.br.
} 
describes an avoidance test used to assess soil quality and toxicity from chemical to Eisenia fetida (earthworm). This test can be applied as a rapid screening test to determinate the bioavailability of chemicals or contaminants in soil to E. fetida, where the avoidance behaviour is used as indicator. In accordance to the results, it can be decided if reproduction test with the same species must be performed. Samples from contaminated sites with different hydrocarbons concentrations collected from a bus garage, a gas station and a contaminated oil spilling area were analysed. The results of the sample from the bus station showed that $96 \%$ of the organisms escaped from the contaminated sample section to the control soil section. This indicates that the sample can be considered toxic, presenting the habitat function limited. The toxicity of this sample was confirmed later by mortality and reproduction tests. The preliminary results indicated that avoidance test was a rapid indicator of sample toxicity and can be applied as a complement to evaluate hydrocarbons contaminated sites.

Key words: terrestrial ecotoxicology, earthworm, Eisenia fetida, contaminated sites, hydrocarbons.

\section{INTRODUÇÃO}

Os problemas oriundos de áreas contaminadas vêm crescendo cada vez mais no Brasil. Essas áreas podem ser representadas por postos de serviços e abastecimento, locais de disposição inadequada de resíduos sólidos, indústrias desativadas, etc., e muitas vezes encontram-se nas proximidades de núcleos populacionais e ecossistemas, podendo causar impactos negativos ao ambiente e à saúde.

Estudos de avaliação de risco ambiental, ecológico e à saúde humana podem ser realizados em áreas contaminadas, mas ainda são considerados em desenvolvimento e pouco aplicados no Brasil (Sisinno, 2003).

Algumas áreas contaminadas por substâncias químicas de importância para a saúde humana e ambiental têm sido freqüentemente estudadas e cresce o interesse dos órgãos de saúde e ambiente no controle do impacto causado por esses locais, uma vez que a identificação e o cadastramento dessas áreas permitem que ações preventivas ou corretivas relacionadas à possível contaminação do solo, ar, águas superficiais e subterrâneas e biota sejam estabelecidas, e os riscos minimizados.

A avaliação da contaminação dessas áreas tem sido realizada apenas com o auxílio de parâmetros químicos, sem a inserção da avaliação ecotoxicológica. Entretanto, por meio dos ensaios ecotoxicológicos são verificados os efeitos das variáveis ambientais que são capazes de afetar a toxicidade das substâncias aos componentes vivos de um ecossistema. Dessa forma, esses ensaios podem indicar uma resposta mais precisa da toxicidade dos contaminantes presentes nas amostras para os organismos vivos; o que apenas a análise química de cada composto, separadamente, não é capaz de avaliar.

Os procedimentos para execução de ensaios ecotoxicológicos com organismos aquáticos são bem estabelecidos e descritos em normas técnicas brasileiras da Associação Brasileira de Normas Técnicas (ABNT), métodos de alguns órgãos de fiscalização ambiental, como a Fundação Estadual de Engenharia do Meio Ambiente - RJ (FEEMA) e a Companhia de Tecnologia de Saneamento Ambiental - SP (CETESB), e métodos descritos no Manual de Testes para Avaliação da Ecotoxicidade de Agentes Químicos, organizado pelo Instituto Brasileiro de Meio Ambiente e dos Recursos Naturais Renováveis (IBAMA) (Sisinno et al., 2004).
Entretanto, os ensaios para avaliação da ecotoxicidade com organismos de solo não estão bem estabelecidos no Brasil por não existirem ainda normas da ABNT e pelo fato de os métodos descritos no Manual do IBAMA estarem desatualizados. Dessa forma, quando há necessidade de avaliação da contaminação de amostras de solos, normalmente são utilizados métodos internacionalmente reconhecidos como os da ISO (International Organization for Standardization), OECD (Organization for Economic Co-operation and Development) e EPA (Environmental Protection Agency - USA) (Sisinno et al., 2004).

Esses métodos, entretanto, são desenvolvidos para determinar a toxicidade de substâncias adicionadas a um solo artificial, a fim de que vários interferentes sejam eliminados. $\mathrm{O}$ grande desafio na adaptação desses métodos para a complementação da avaliação de áreas contaminadas é a substituição do substrato artificial pelas amostras de solos trazidas dessas áreas, a avaliação dos possíveis interferentes nos resultados, bem como a escolha dos organismos-teste para amostras com determinadas características.

O objetivo deste estudo foi realizar ensaios de toxicidade com minhocas em amostras naturais provenientes de áreas contaminadas por hidrocarbonetos, a fim de que sua utilização seja analisada como complementação no processo de avaliação de áreas contaminadas.

\section{MATERIAIS E MÉTODOS}

Amostras de áreas contaminadas por hidrocarbonetos originadas de uma garagem de ônibus (Amostra 1), um posto de serviços e abastecimento (Amostra 2) e uma área com derramamento de óleo (Amostra 3) foram avaliadas utilizandose ensaios de comportamento com minhocas da espécie Eisenia fetida (Savigny, 1826) (Oligochaeta, Lumbricidae), segundo $\mathrm{o}$ draft da ISO Avoidance test for testing the quality of soils and the toxicity of chemicals (ISO, 2002).

Os ensaios de letalidade e reprodução foram realizados de acordo com as normas ISO 11268-1 - Soil quality - Effects of pollutants on earthworms (Eisenia fetida) - Part 1: Determination of acute toxicity using artificial soil substrate (ISO, 1993) e ISO 11268-2 - Soil quality - Effects of pollutants on earthworms (Eisenia fetida) - Part 2: Determination of effects on reproduction (ISO, 1998). 
O solo controle foi preparado artificialmente, segundo a ISO 11268-2, na proporção de 70\% de areia lavada e peneirada, $20 \%$ de caulim em pó e $10 \%$ de Sphagnum (briófita) moído. Todos os ensaios foram realizados em triplicata, sendo desenvolvidos em condições controladas de temperatura $\left(20^{\circ} \mathrm{C} \pm 2^{\circ} \mathrm{C}\right)$, luminosidade (400 lux a 800 lux) e fotoperíodo (12 h: $12 \mathrm{~h}$ ).

$\mathrm{O}$ lote dos organismos utilizados foi avaliado por meio de um ensaio com a substância de referência Cloroacetamida, indicada nas normas da ISO para o organismo-teste usado. A CL(I)50 da Cloroacetamida deve estar entre $20 \mathrm{mg} / \mathrm{kg}$ e 80 $\mathrm{mg} / \mathrm{kg}$ (ISO, 1993). As minhocas utilizadas foram indivíduos adultos, com clitelo desenvolvido e peso entre 300 e $600 \mathrm{~g}$.

O ensaio de comportamento, ainda pouco testado no Brasil, pode ser aplicado como um método rápido para determinar a biodisponibilidade de substâncias químicas ou de contaminantes no solo para E. fetida, em que o comportamento de fuga é usado como indicador (ISO, 2002).

O princípio desse ensaio é a exposição das minhocas simultaneamente às amostras de solo não contaminado (controle) e contaminado. Ambas as amostras são colocadas no mesmo recipiente, em seções distintas, separadas por meio de uma divisória, formando dois compartimentos. Após a arrumação das amostras em cada lado da divisória, a mesma é retirada, formando uma linha onde 10 organismos são colocados na superfície. Ao final de 48 horas, o número de organismos é determinado em cada seção do recipiente. A amostra é considerada tóxica (com a função de habitat do solo limitada) se forem encontrados mais de $80 \%$ do total de organismos expostos na amostra do solo controle.

No início do ensaio de letalidade foram colocados 10 organismos adultos em cada recipiente com a amostra contaminada. Após o 7ํ dia de exposição, os organismos mortos foram retirados e os sobreviventes mantidos até o $14^{\circ}$ dia, quando, então, foram separados para que o porcentual de mortalidade obtido fosse comparado com o controle.

No ensaio de reprodução, os 10 organismos permaneceram nos recipientes até o $28^{\circ}$ dia, quando foram retirados para que apenas os casulos resultantes permanecessem até o $56^{\circ}$ dia do ensaio. Após esse período, os jovens foram contados e comparados ao controle.

\section{RESULTADOS E DISCUSSÃO}

Os resultados preliminares para o ensaio de comportamento mostraram que na Amostra 1, proveniente de uma garagem de ônibus, $96 \%$ dos organismos foram encontrados vivos na seção que continha o solo controle, indicando que esta amostra pode ser considerada tóxica, apresentando sua função de habitat limitada.

As Amostras 2 e 3 não foram consideradas tóxicas pelo ensaio de comportamento, uma vez que $33 \%$ e $63 \%$ dos organismos, respectivamente, foram encontrados na seção onde estava o solo controle. Dessa forma, procedeu-se aos ensaios de letalidade e reprodução apenas com a Amostra 1.

No ensaio de letalidade (toxicidade aguda), os 30 organismos expostos ao solo contaminado morreram, indicando alta mortalidade (100\%) quando comparada ao controle $(0 \%$ de morte).

Em relação à taxa de reprodução, não foram encontrados casulos nem indivíduos jovens na amostra avaliada, enquanto no controle foram encontrados 21 casulos e 30 organismos jovens.

Uma vez que não houve necessidade de ajustes nas características da Amostra 1 para garantir a sobrevivência dos organismos durante os ensaios (apenas a umidade foi ajustada para aproximadamente $60 \%$, conforme exigência das condições para os ensaios), entende-se que os resultados obtidos estejam expressando de forma bem realista o impacto negativo da contaminação dessa área para um importante organismo do solo, como é o caso da minhoca.

Os resultados preliminares mostram que o ensaio de comportamento foi um indicador rápido da toxicidade, uma vez que tanto o ensaio de letalidade como o de reprodução mostraram, após maior período de exposição, a alta toxicidade da amostra.

Dessa forma, entende-se que os ensaios realizados com $E$. fetida - adaptados com o uso de solos de áreas contaminadas podem ser aplicados na complementação da avaliação de áreas contaminadas por hidrocarbonetos. De acordo com os resultados de Hund-Rinke \& Wiechering (2001), os ensaios de comportamento com amostras de áreas contaminadas mostraram significativamente maior sensibilidade do que os ensaios com amostras contaminadas artificialmente.

Entretanto, deve-se ressaltar que variáveis relacionadas à composição do solo a ser testado precisam ser sempre bem avaliadas, se o objetivo dos ensaios for a avaliação da toxicidade com amostras naturais, sem a necessidade de mistura com o solo artificial.

Esses ensaios poderão ser aplicados na avaliação de risco ecológico para verificar o grau de injúria aos organismos representantes do ecossistema afetado e sua capacidade de encontrar condições de se estabelecer novamente em uma área comprometida por contaminação química.

Além disso, esses ensaios também poderão auxiliar na avaliação da capacidade de recuperação ecológica de uma área, após processo de descontaminação. Atualmente, a efetiva descontaminação de uma área é avaliada apenas por análises químicas. Entretanto, não se pode afirmar que as concentrações remanescentes dos contaminantes ainda não sejam tóxicas para os organismos do solo ou mesmo que o uso de substâncias durante o processo de remediação da área não possa ter afetado seu equilíbrio ecológico. 
Agradecimentos - Os autores gostariam de agradecer o apoio fundamental dos seguintes profissionais: Regina Sáfadi e Alice Fontes da TECAM; Marcos Garcia da EMBRAPA-Manaus; Daniele Alves da Rocha da FEEMA e Jorge Luiz Florindo da Cruz do CETEM.

\section{REFERÊNCIAS BIBLIOGRÁFICAS}

HUND-RINKE, K. \& WIECHERING, H., 2001, Earthworm avoidance test for soil assessments: an alternative for acute and reproduction tests. $J$. Soils \& Sediments, 1: 15-20.

ISO (INTERNATIONAL ORGANIZATION FOR STANDARDIZATION), 1993, ISO 11268-1. Soil quality - Effects of polutants on earthworms (Eisenia fetida) - Part 1: determination of acute toxicity using artificial soil substrate. Geneva, ISO.

ISO (INTERNATIONAL ORGANIZATION FOR STANDARDIZATION), 1998, ISO 11268-2. Soil quality - Effects of polutants on earthworms (Eisenia fetida) - Part 2: determination of effects on reproduction. Geneva, ISO.
ISO (INTERNATIONAL ORGANIZATION FOR STANDARDIZATION), 2002, Draft. Avoidance test for testing the quality of soils and the toxicity of chemicals - Part 1: test with earthworms (Eisenia fetida). Geneva, ISO.

SISINNO, C. L. S., 2003, Uso de ensaios ecotoxicológicos com organismos aquáticos e do solo para avaliar a toxicidade de amostras de solos contaminados e resíduos sólidos. Proposta de projeto de pesquisa da FIOCRUZ, Rio de Janeiro.

SISINNO, C., BULUS, M., RIZZO, A., SÁFADI, R., FONTES, A. \& MOREIRA, J., 2004, Ensaios ecotoxicológicos como um instrumento de complementação da avaliação de áreas contaminadas: resultados preliminares em áreas contaminadas por hidrocarbonetos, pp. 150-154. In: III Seminário Nacional de Saúde e Ambiente, Fundação Oswaldo Cruz, Rio de Janeiro, 164p. 